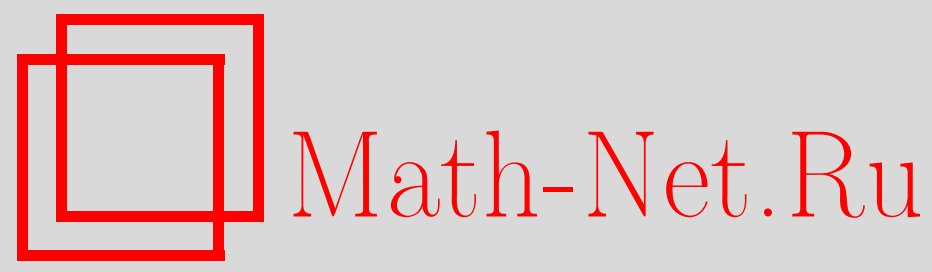

Р. Н. Бояринов, О числе промежутков Грама, содержащих ординаты последовательных нулей дзета-функции Римана, Дискрет. матем., 2012, том 24, выпуск 3, 152-159

DOI: https://doi.org/10.4213/dm1205

Использование Общероссийского математического портала Math-Net.Ru подразумевает, что вы прочитали и согласны с пользовательским соглашением http://www. mathnet.ru/rus/agreement

Параметры загрузки:

IP: 54.237 .59 .107

26 апреля 2023 г., 13:54:31 


\title{
О числе промежутков Грама, содержащих ординаты последовательных нулей дзета-функции Римана
}

\author{
(c) 2012 г. Р. Н. Бояринов
}

Получена верхняя оценка числа промежутков Грама, содержащих $k$ ординат последовательных нулей дзета-функции Римана.

При $t>0$ определим функцию $\vartheta(t)$ как приращение непрерывной ветви аргумента функции $\pi^{-s / 2} \Gamma(s / 2)$ при изменении $s$ вдоль отрезка, соединяющего точки $s=1 / 2$ и $s=1 / 2+i t$. Выбрав ветвь аргумента, значение которой в точке $s=1 / 2$ равно нулю, получаем

$$
\vartheta(t)=\operatorname{Im} \ln \Gamma\left(\frac{1}{4}+\frac{i t}{2}\right)-\frac{t}{2} \ln \pi=\frac{t}{2} \ln \frac{t}{2 \pi}-\frac{t}{2}-\frac{\pi}{8}+\Delta(t),
$$

где

$$
\begin{aligned}
& \Delta(t)=\frac{t}{4} \ln \left(1+\frac{1}{4 t^{2}}\right)+\frac{1}{4} \operatorname{arctg}\left(\frac{1}{2 t}\right)-\frac{t}{2} \int_{0}^{\infty} \frac{\rho(u) d u}{(u+1 / 4)^{2}+t^{2} / 4}, \\
& \rho(u)=1 / 2-\{u\} .
\end{aligned}
$$

Пусть $n \geqslant 0$ - целое число. Назовем точкой Грама $g_{n}$ единственный корень уравнения

$$
\vartheta\left(g_{n}\right)=\pi(n-1)
$$

а $n$-м промежутком Грама $G_{n}-$ промежуток $\left(g_{n-1}, g_{n}\right]$. При $n \rightarrow \infty$ справедливы асимптотические формулы

$$
g_{n}=\frac{2 \pi n}{\ln n}(1+o(1)), \quad g_{n+1}-g_{n}=\frac{2 \pi}{\ln n}(1+o(1)) .
$$

В 1903 г. Дж. Грам [1] установил, что первые 15 промежутков $G_{n}$ содержат только по одному нулю функции $\zeta(1 / 2+i t)$. Иными словами, первые пятнадцать нулей функции $\zeta(1 / 2+i t)$ отделены друг от друга точками Грама. Грам предположил, что обнаруженная закономерность не является общей.

В 1925 г. Дж. Хатчинсон [2] нашел два исключения: промежуток $G_{127}$ не содержал ни одного нуля функции $\zeta(1 / 2+i t)$, а промежуток $G_{135}$ содержал даже два нуля.

Тем не менее, в большинстве рассмотренных случаев каждый промежуток Грама содержал ровно один нуль функции $\zeta(1 / 2+i t)$. Свойство нулей функции $\zeta(1 / 2+i t)$ быть отделенными точками Грама было названо правилом Грама (законом Грама). 
В 1934 г. Е. Титчмарш [3] получил оценку снизу для количества промежутков Грама, лежащих на $(0, T)$ и содержащих не менее одного нуля функции $\zeta(1 / 2+i t)$. Тем самым Е. Титчмарш доказал, что бесконечно много промежутков Грама содержат по крайней мере один нуль функции $\zeta(1 / 2+i t)$.

В 1946 г. А. Сельберг [4] доказал существование положительных постоянных $K$ и $N_{0}$ таких, что для любого $N>N_{0}$ среди первых $N$ промежутков Грама найдется не менее $K N$ промежутков $G_{n}$, содержащих не менее одного нуля функции $\zeta(1 / 2+i t)$ и не менее $K N$ промежутков $G_{n}$, не содержащих ни одного нуля функции $\zeta(1 / 2+i t)$.

В 1977 г. Я. Мозер [5] получил оценку снизу для количества промежутков Грама, лежащих на $(T, T+H]$ и содержащих хотя бы один нуль $\zeta(1 / 2+i t), H=T^{1 / 2} \psi(T) \ln T$, где $\psi(T)$ - возрастающая к бесконечности функция, уточнив тем самым результат Е. Титчмарша.

В данной статье доказывается оценка сверху для числа промежутков Грама, лежащих на коротком интервале и содержащих $k$ ординат последовательных нулей дзета-функции Римана из критической полосы.

Обозначим через $v_{k}(N)$ количество промежутков Грама $G_{n}$ с номерами $n \leqslant N$, содержащих $k$ ординат последовательных (одинаковые ординаты различных нулей получают различные номера) нулей дзета-функции Римана из критической полосы.

Далее $S(t)$ - аргумент дзета-функции Римана на критической прямой (определение см. в [7]). Справедлива следующая теорема.

Теорема 1. Пусть $0<\varepsilon<0,001, N>N_{0}(\varepsilon)>0, M=\left[N^{27 / 82+\varepsilon}\right]$. Тогда для любого иелого $k \geqslant 4 / \varkappa$ справедливо неравенство

$$
\sum_{j=k}^{\infty}\left(v_{j}(N+M)-v_{j}(N)\right) \leqslant \frac{e^{2,1} M}{15} \exp (-\varkappa k),
$$

где $\varkappa=2 \varepsilon^{3 / 2} /\left(3 e^{18}\right)$.

Замечание 1. Неравенство теоремы с $M=\left[N^{\varepsilon}\right]$ верно для всех $N \in(X, 2 X), X \geqslant X_{0}(\varepsilon)$, за исключением значений, образующих множество $E$ с мерой $\operatorname{mes}(E)<X^{1-0,04 \varepsilon}$.

Для доказательства теоремы 1 нам потребуется ряд вспомогательных утверждений.

Лемма 1. Пусть $0<\varepsilon<0,001, N>N_{0}(\varepsilon)>0, M=\left[N^{27 / 82+\varepsilon}\right], x=g_{N}^{0,1 \varepsilon}, m-$ иелое число, $1 \leqslant m<(\ln x) / 192, x^{1 /(4 m)}<y \leqslant x^{1 / m}$. Тогда имеет место оценка

$$
\sum_{N<n \leqslant N+M}\left(S\left(g_{n}\right)+\frac{1}{\pi} \sum_{p<y} \frac{\sin \left(g_{n} \ln p\right)}{\sqrt{p}}\right)^{2 m}<\frac{1}{20}\left(A m^{2}\right)^{m} M,
$$

где $A=e^{34} \varepsilon^{-3}$.

Доказательство леммы можно найти в [6], см. с. 98.

Замечание 2. Неравенство леммы 1 с $M=\left[N^{\varepsilon}\right]$ верно для всех $N \in(X, 2 X), X \geqslant X_{0}(\varepsilon)$, за исключением значений, образующих множество $E$ с мерой $\operatorname{mes}(E)<X^{1-0,04 \varepsilon}$.

Следствие 1. При выполнении условий леммы 1 справедлива оценка

$$
\sum_{N<n \leqslant N+M}\left(S\left(g_{n+1}\right)+\frac{1}{\pi} \sum_{p<y} \frac{\sin \left(g_{n+1} \ln p\right)}{\sqrt{p}}\right)^{2 m}<\frac{1}{10}\left(A m^{2}\right)^{m} M,
$$

где $A=e^{34} \varepsilon^{-3}$. 
Доказательство. Пусть

$$
\begin{aligned}
A_{m} & =\sum_{N<n \leqslant N+M}\left(S\left(g_{n+1}\right)+\frac{1}{\pi} \sum_{p<y} \frac{\sin \left(g_{n+1} \ln p\right)}{\sqrt{p}}\right)^{2 m}, \\
B_{m} & =\sum_{N<n \leqslant N+M}\left(S\left(g_{n}\right)+\frac{1}{\pi} \sum_{p<y} \frac{\sin \left(g_{n} \ln p\right)}{\sqrt{p}}\right)^{2 m}, \\
C_{m} & =\left(S\left(g_{N+M+1}\right)+\frac{1}{\pi} \sum_{p<y} \frac{\sin \left(g_{N+M+1} \ln p\right)}{\sqrt{p}}\right)^{2 m} .
\end{aligned}
$$

Тогда

$$
A_{m} \leqslant B_{m}+C_{m}
$$

Докажем, что

$$
C_{m} \leqslant \frac{1}{20}\left(A m^{2}\right)^{m} M
$$

Отсюда будет следовать справедливость следствия 1. Так как (см. [7], с. 73) верно неравенство

$$
\mid S\left(g_{N+M+1} \mid \leqslant 15,5 \ln g_{N+M+1}<16 \ln N,\right.
$$

то

$$
C_{m} \leqslant(16 \ln N+\sqrt{y})^{2 m} \leqslant 2^{2 m}\left(16^{2 m} \ln ^{2 m} N+x\right) .
$$

Верны неравенства

$$
4^{m} x \leqslant 4^{m} N^{0,1 \varepsilon}<\frac{1}{64}\left(A m^{2}\right)^{m} M, \quad(32 \ln N)^{2 m} \leqslant \frac{1}{32^{m}}\left(A m^{2}\right)^{m} M .
$$

Последнее неравенство эквивалентно

$$
32^{3} \ln ^{2} N \leqslant A m^{2} M^{1 / m} .
$$

Функция

$$
f(t)=t^{2} M^{1 / t}
$$

на промежутке $[1, \infty)$ принимает минимальное значение в точке $t=(\ln M) / 2$. Так как $M \geqslant\left[N^{\varepsilon}\right]>N^{\varepsilon / 2}$, то

$$
\frac{\ln M}{2}>\frac{\varepsilon}{4} \ln N>\frac{0,1 \varepsilon}{192} \ln N>m \geqslant 1 .
$$

Отсюда имеем

$$
f(m) \geqslant f\left(\frac{1}{2} \ln M\right)=\frac{e^{2}}{4} \ln ^{2} M>\ln ^{2} M .
$$

Получаем

$$
A m^{2} M^{1 / m}>A \ln ^{2} M>\frac{A \varepsilon^{2}}{4} \ln ^{2} N>32^{3} \ln ^{2} N .
$$

Отсюда

$$
C_{m} \leqslant \frac{3}{64}\left(A m^{2}\right)^{m} M<\frac{1}{20}\left(A m^{2}\right)^{m} M .
$$


Замечание 3. Неравенство следствия 1 с $M=\left[N^{\varepsilon}\right]$ верно для всех $N \in(X, 2 X)$, $X \geqslant X_{0}(\varepsilon)$, за исключением значений, образующих множество $E$ с мерой $\operatorname{mes}(E)<X^{1-0,04 \varepsilon}$.

Лемма 2. Пусть $0<\varkappa<1 / 2,0<c<1 / 2-\varkappa, k, r, v, N, M-$ иелье числа, $k \geqslant 0$, $r \geqslant 0, k+r=v, v \geqslant 1, N \geqslant \exp \left(9 \varkappa^{-1}\right), M \geqslant \exp \left(\frac{3}{2} v c^{-1}\right), M \leqslant N, y=M^{2 v / c}$. Пyсть, далее, $p_{1}, \ldots, p_{k}, q_{1}, \ldots, q_{r}$ принимают значения простых чисел из промежутка $(1 ; y] u$ удовлетворяют условию $p_{1} \cdots p_{k} \neq q_{1} \cdots q_{r}$. Пусть, наконеи, все члены последовательности $а(p)$ при $p \leqslant y$ подчинены условию $|a(p)| \leqslant \delta$. Если $g_{n}-$ последовательность точек Грама, то для суммы

$$
\Sigma=\sum_{N<n \leqslant N+M} \sum_{\substack{p_{1}, \ldots, p_{k} \\ q_{1}, \ldots, q_{r}}} \frac{a\left(p_{1}\right) \cdots a\left(p_{k}\right) \bar{a}\left(q_{1}\right) \cdots \bar{a}\left(q_{r}\right)}{\left(p_{1} \cdots p_{k} q_{1} \cdots q_{r}\right)^{1 / 2}}\left(\frac{q_{1} \cdots q_{r}}{p_{1} \cdots p_{k}}\right)^{i g_{n}}
$$

имеет место оиенка

$$
|\Sigma|<\left(\delta y^{3 / 2}\right)^{v} \ln N .
$$

Доказательство леммы можно найти в [6], см. с. 92.

Лемма 3. Пусть $g_{n}-$ точка Грама с номером $N<n \leqslant N+M$, где $N, M-$ натуральные числа, $M<N$, и

$$
\begin{aligned}
h_{n} & =\frac{g_{n+1}-g_{n}}{2}, & h & =\frac{\pi}{\ln \left(g_{N} /(2 \pi)\right)}, \\
b_{n}(p) & =p^{i h_{n}} \sin \left(h_{n} \ln p\right), & a(p) & =p^{i h} \sin (h \ln p) .
\end{aligned}
$$

Тогда справедливы равенства

$$
h_{n}=h+O\left(\frac{M}{N \ln ^{2} N}\right), \quad b_{n}(p)=a(p)+O\left(\frac{M}{N \ln N}\right),
$$

где постоянные под знаком $О$ абсолютные.

Доказательство. Из формулы Лагранжа о конечных приращения для функции $\vartheta(t)$ получаем

$$
\begin{aligned}
g_{n+1}-g_{n} & =\frac{\vartheta\left(g_{n+1}\right)-\vartheta\left(g_{n}\right)}{\vartheta^{\prime}\left(\xi_{n}\right)}=\frac{\pi}{\vartheta^{\prime}\left(\xi_{n}\right)} \\
& =\frac{2 \pi}{\ln \left(\xi_{n} /(2 \pi)\right)+O\left(1 / \xi_{n}^{2}\right)}, \quad g_{n}<\xi_{n}<g_{n+1} .
\end{aligned}
$$

Отсюда

$$
\begin{aligned}
g_{n+1}-g_{n} & =\frac{2 \pi}{\ln \left(\xi_{n} /(2 \pi)\right)}+O\left(\frac{1}{\xi_{n}^{2} \ln \xi_{n}}\right) \\
& =\frac{2 \pi}{\ln \left(\xi_{n} /(2 \pi)\right)}+O\left(\frac{1}{g_{n}^{2} \ln g_{n}}\right) \\
& =\frac{2 \pi}{\ln \left(\xi_{n} /(2 \pi)\right)}+O\left(\frac{1}{N^{2}}\right) .
\end{aligned}
$$


Далее,

$$
\begin{aligned}
\ln \left(\frac{\xi_{n}}{2 \pi}\right) & =\ln \left(\frac{g_{N}}{2 \pi}\right)+O\left(\ln \frac{g_{N+M+1}}{g_{N}}\right) \\
& =\ln \left(\frac{g_{N}}{2 \pi}\right)+O\left(\frac{M}{N}\right) .
\end{aligned}
$$

Поэтому

$$
g_{n+1}-g_{n}=\frac{2 \pi}{\ln \left(g_{N} /(2 \pi)\right)}+O\left(\frac{M}{N \ln ^{2} N}\right) .
$$

Отсюда следуют утверждения леммы 3.

Лемма 4. Пусть $0<\varepsilon<0,001, N>N_{0}(\varepsilon)>0, M=\left[N^{27 / 82+\varepsilon}\right], x=g_{N}^{0,1 \varepsilon}, m-$ ичелое число, $1 \leqslant m<(\ln x) / 192$. Тогда верно неравенство

$$
\sum_{N<n \leqslant N+M}\left(S\left(g_{n+1}\right)-S\left(g_{n}\right)\right)^{2 m}<\frac{1}{15}\left(9 A m^{2}\right)^{m} M,
$$

где $A=e^{34} \varepsilon^{-3}$.

Доказательство. Пусть

$$
\begin{aligned}
h_{n} & =\frac{g_{n+1}-g_{n}}{2}, & h & =\frac{\pi}{\ln \left(g_{N} /(2 \pi)\right)}, \\
b_{n}(p) & =p^{i h_{n}} \sin \left(h_{n} \ln p\right), & a(p) & =p^{i h} \sin (h \ln p), \\
U_{n} & =\frac{1}{\pi} \sum_{p<y} b_{n}(p) p^{-1 / 2+i g_{n}}, & x^{1 /(4 m)} & <y \leqslant x^{1 / m} .
\end{aligned}
$$

Тогда

$$
\begin{aligned}
S\left(g_{n+1}\right)-S\left(g_{n}\right)=\left(S\left(g_{n+1}\right)+\frac{1}{\pi} \sum_{p<y}\right. & \left.\frac{\sin \left(g_{n+1} \ln p\right)}{\sqrt{p}}\right) \\
& -\left(S\left(g_{n}\right)+\frac{1}{\pi} \sum_{p<y} \frac{\sin \left(g_{n} \ln p\right)}{\sqrt{p}}\right)-\left(U_{n}+\bar{U}_{n}\right) .
\end{aligned}
$$

Используя неравенство Гельдера, получаем, что

$$
\sum_{N<n \leqslant N+M}\left(S\left(g_{n+1}\right)-S\left(g_{n}\right)\right)^{2 m} \leqslant 3^{2 m-1}\left(A_{m}+B_{m}+D_{m}\right),
$$

где $A_{m}, B_{m}$ определены в доказательстве следствия 1 и

$$
D_{m}=\sum_{N<n \leqslant N+M}\left(U_{n}+\bar{U}_{n}\right)^{2 m} .
$$

Из леммы 1 и следствия 1 получаем, что

$$
A_{m}+B_{m} \leqslant \frac{3}{20}\left(A m^{2}\right)^{m} M .
$$


Докажем, что для $D_{m}$ выполняется неравенство

$$
D_{m} \leqslant \frac{1}{20}\left(A m^{2}\right)^{m} M
$$

Действительно,

$$
D_{m}=\sum_{k=0}^{2 m}\left(\begin{array}{c}
2 m \\
k
\end{array}\right) \sigma_{k}
$$

где $\left(\begin{array}{c}2 m \\ k\end{array}\right)-$ биномиальный коэффициент, а

$$
\sigma_{k}=\frac{1}{\pi^{2 m}} \sum_{N<n \leqslant N+M} \sum_{\substack{p_{1}, \ldots, p_{k} \\ q_{1}, \ldots, q_{2 m-k}}} \frac{b_{n}\left(p_{1}\right) \cdots b_{n}\left(p_{k}\right) \bar{b}_{n}\left(q_{1}\right) \cdots \bar{b}_{n}\left(q_{2 m-k}\right)}{\left(p_{1} \cdots p_{k} q_{1} \cdots q_{2 m-k}\right)^{1 / 2}}\left(\frac{p_{1} \cdots p_{k}}{q_{1} \cdots q_{2 m-k}}\right)^{i g_{n}} .
$$

Используя лемму 3 , получаем, что

$$
\sigma_{k}=\omega_{k}+O\left(\frac{1}{\pi^{2 m}} \frac{M^{2} y^{m}}{N}\right)=\omega_{k}+O\left(\frac{1}{\pi^{2 m}} \frac{1}{N^{1 / 4}}\right),
$$

где

$$
\omega_{k}=\frac{1}{\pi^{2 m}} \sum_{N<n \leqslant N+M} \sum_{\substack{p_{1}, \ldots, p_{k} \\ q_{1}, \ldots, q_{2 m-k}}} \frac{a\left(p_{1}\right) \cdots a\left(p_{k}\right) \bar{a}\left(q_{1}\right) \cdots \bar{a}\left(q_{2 m-k}\right)}{\left(p_{1} \cdots p_{k} q_{1} \cdots q_{2 m-k}\right)^{1 / 2}}\left(\frac{p_{1} \cdots p_{k}}{q_{1} \cdots q_{2 m-k}}\right)^{i g_{n}} .
$$

При $k \neq m$ из леммы 2 получаем оценку для $\omega_{k}$ :

$$
\left|\omega_{k}\right| \leqslant \frac{y^{3 m} \ln N}{\pi^{2 m}} \leqslant \frac{x^{3} \ln N}{\pi^{2 m}}<\frac{N^{0,3 \varepsilon}}{\pi^{2 m}} .
$$

Отсюда получаем, что

$$
\left|\sigma_{k}\right| \leqslant \frac{0,6 M}{\pi^{2 m}}
$$

Далее,

$$
\begin{aligned}
\omega_{m}= & \frac{M}{\pi^{2 m}} \sum_{p_{1} \cdots p_{m}=q_{1} \cdots q_{m}} \frac{a\left(p_{1}\right) \cdots a\left(p_{m}\right) \bar{a}\left(q_{1}\right) \cdots \bar{a}\left(q_{m}\right)}{\left(p_{1} \cdots p_{m} q_{1} \cdots q_{m}\right)^{1 / 2}} \\
& +\frac{1}{\pi^{2 m}} \sum_{N<n \leqslant N+M} \sum_{p_{1} \cdots p_{m} \neq q_{1} \cdots q_{m}} \frac{a\left(p_{1}\right) \cdots a\left(p_{m}\right) \bar{a}\left(q_{1}\right) \cdots \bar{a}\left(q_{m}\right)}{\left(p_{1} \cdots p_{m} q_{1} \cdots q_{m}\right)^{1 / 2}}\left(\frac{p_{1} \cdots p_{m}}{q_{1} \cdots q_{m}}\right)^{i g_{n}} \\
= & \omega_{m}^{*}+\omega_{m}^{* *} .
\end{aligned}
$$

Из леммы 2 получаем оценку для $\omega_{m}^{* *}$ :

$$
\left|\omega_{m}^{* *}\right| \leqslant \frac{y^{3 m} \ln N}{\pi^{2 m}} \leqslant \frac{x^{3} \ln N}{\pi^{2 m}}<\frac{N^{0,3 \varepsilon}}{\pi^{2 m}}<\frac{0,1 M}{\pi^{2 m}} .
$$

Используя однозначность разложения натурального числа, большего единицы, на простые 
сомножители, для $\omega_{m}^{*}$ получаем следующую оценку:

$$
\begin{aligned}
\left|\omega_{m}^{*}\right| & \leqslant \frac{M}{\pi^{2 m}} \sum_{p_{1} \cdots p_{m}=q_{1} \cdots q_{m}} \frac{\left|a\left(p_{1}\right)\right| \cdots\left|a\left(p_{m}\right)\right|\left|\bar{a}\left(q_{1}\right)\right| \cdots\left|\bar{a}\left(q_{m}\right)\right|}{\left(p_{1} \cdots p_{m} q_{1} \cdots q_{m}\right)^{1 / 2}} \leqslant \frac{M m !}{\pi^{2 m}}\left(\sum_{p<y} \frac{|a(p)|^{2}}{p}\right)^{m} \\
& =\frac{M m !}{\pi^{2 m}}\left(\sum_{p<y} \frac{\sin ^{2}(h \ln p)}{p}\right)^{m} \leqslant \frac{M m !}{\pi^{2 m}}\left(h^{2} \ln y \sum_{p<y} \frac{\ln p}{p}\right)^{m} \\
& \leqslant \frac{M m !}{\pi^{2 m}}(2 h \ln y)^{2 m}<\frac{M}{\pi^{2 m}}\left(\frac{2 \pi \varepsilon}{5 \sqrt{m}}\right)^{2 m}<\frac{0,1 M}{\pi^{2 m}} .
\end{aligned}
$$

Отсюда получаем, что

$$
\left|\sigma_{m}\right|<\frac{0,3 M}{\pi^{2 m}}
$$

Далее,

$$
\left|D_{m}\right|<\left(\begin{array}{c}
2 m \\
m
\end{array}\right)\left|\sigma_{m}\right|+\sum_{\substack{k=0 \\
k \neq m}}^{2 m}\left(\begin{array}{c}
2 m \\
k
\end{array}\right)\left|\sigma_{k}\right|<\frac{2^{2 m}}{\pi^{2 m}} 0,9 M<\frac{1}{20}\left(A m^{2}\right)^{m} M .
$$

Отсюда следует справедливость леммы 4.

Замечание 4. Неравенство леммы 4 с $M=\left[N^{\varepsilon}\right]$ верно для всех $N \in(X, 2 X), X \geqslant X_{0}(\varepsilon)$, за исключением значений, образующих множество $E$ с мерой $\operatorname{mes}(E)<X^{1-0,04 \varepsilon}$.

Доказательство теоремь 1. Количество $N(T)$ нулей дзета-функции Римана $\zeta(s)$, удовлетворяющих условию $0<\operatorname{Im} s \leqslant T, 0 \leqslant \operatorname{Re} s \leqslant 1$, выражается формулой РиманаМангольдта (см. [7], с. 44):

$$
N(T)=\frac{\vartheta(t)}{\pi}+1+S(T) .
$$

Перенумеруем мнимые части нулей $\zeta(s)$ в критической полосе в порядке возрастания, а в случае совпадения нескольких ординат - в произвольном порядке следующим образом: $0<\gamma_{1}<\gamma_{2}<\ldots \leqslant \gamma_{n} \leqslant \gamma_{n+1} \leqslant \ldots$ Пусть промежуток $\left(g_{n}, g_{n+1}\right]$ содержит только ординаты $\gamma_{s+1}, \ldots, \gamma_{s+l}$, где $l \geqslant k$. Тогда

$$
S\left(g_{n+1}\right)-S\left(g_{n}\right)=N\left(g_{n+1}\right)-N\left(g_{n}\right)-1 \geqslant N\left(g_{n+1}\right)-N\left(g_{n}+0\right)-1 \geqslant k-3 / 2 .
$$

Далее,

$$
(k-3 / 2)^{2 m} \sum_{j=k}^{\infty}\left(v_{j}(N+M)-v_{j}(N)\right) \leqslant \sum_{N<n \leqslant N+M}\left(S\left(g_{n+1}\right)-S\left(g_{n}\right)\right)^{2 m} .
$$

Заметим, что при $k \geqslant 32 \ln N$ левая часть этого неравенства будет равна нулю, поскольку нет нулей $\zeta(s)$, ординаты которых лежат на промежутке $\left(g_{N}, g_{N+M}\right]$ и кратность которых больше $32 \ln N$. Применяя лемму 4, получаем, что

$$
(k-3 / 2)^{2 m} \sum_{j=k}^{\infty}\left(v_{j}(N+M)-v_{j}(N)\right)<\frac{1}{15}\left(9 A m^{2}\right)^{m} M .
$$

При $4 / \varkappa \leqslant k \leqslant 32 \ln N$, где $\varkappa=2 \varepsilon^{1.5} /\left(3 e^{18}\right)$, отсюда получаем, что

$$
\sum_{j=k}^{\infty}\left(v_{j}(N+M)-v_{j}(N)\right)<\frac{1}{15}\left(\frac{3 m \sqrt{A}}{k-3 / 2}\right)^{2 m} M .
$$


Возьмем

$$
m=\left[\frac{k-3 / 2}{3 e \sqrt{A}}\right] .
$$

Тогда

$$
\sum_{j=k}^{\infty}\left(v_{j}(N+M)-v_{j}(N)\right)<\frac{1}{15} e^{-2 m} M<\frac{e^{2,1} M}{15} \exp (-\varkappa k) .
$$

Теорема полностью доказана.

\section{Список литературы}

1. Gram J.-P., Note sur les zéros de la fonction $\zeta(s)$ de Riemann. Acta Math. (1903) 27, №1, $289-304$.

2. Hutchinson J. I., On the roots of the Riemann zeta function. Trans. Amer. Math. Soc. (1925) 27, №1, 49-60.

3. Titchmarsh E. C., On van der Corput's method and the zeta-function. IV. Quart. J. Math., Oxf. Ser. (1934) 5, 98-105.

4. Selberg A., The zeta function and the Riemann hypothesis. In: Dixième Congrès Math. Skandinaves 1946, 10. Gjellerups Forlag, Copenhagen, 1947, pp. 187-200.

5. Mózer, J., On Gram's law in the theory of the Riemann zeta-function. Acta Arithmetica (1977) 32, 107-113.

6. Королев М. А., Закон Грама и гипотеза Сельберга о распределении нулей дзета-функции Римана. Известия РАН, сер. матем. (2010) 74, №4, 83-118.

7. Карацуба А. А., Королев М. А., Аргумент дзета-функции Римана. Успехи матем. наук (2005) 60 , №3 (363), 41-96.

8. Карацуба А. А., Королев М. А., Поведение аргумента дзета-функции Римана на критической прямой. Успехи матем. наук (2006) 61, №3 (369), 3-92.

Статья поступила 18.05.2011. 